\title{
EDITORIAL: STATISTICS EDUCATION RESEARCH FROM A LATIN AMERICAN PERSPECTIVE
}

\author{
SPECIAL ISSUE OF THE STATISTICS EDUCATION RESEARCH JOURNAL
}

\section{GUEST EDITORS}

\author{
LEANDRO DE OLIVEIRA SOUZA \\ Universidade Federal De Uberlândia \\ olilean@gmail.com \\ LUCÍA ZAPATA-CARDONA \\ University of Antioquia \\ minervaluka@hotmail.com
PAST SPECIAL ISSUE EDITOR
MANFRED BOROVCNIK
University of Klagenfurt
manfred.borovcnik@aau.at

\author{
MAUREN PORCIÚNCULA \\ Universidade Federal Do Rio Grande \\ mauren@furg.br \\ AUDY SALCEDO \\ Central University of Venezuela \\ audy.salcedo@ucv.ve \\ CURRENT SPECIAL ISSUE EDITOR
DANIEL FRISCHEMEIER
Universität Münster
dfrische@uni-muenster.de
}

A warm welcome to this special issue of the Statistics Education Research Journal (SERJ). This issue is unique as it sets research standards from a specific point of view, namely the Statistics Education Research from a Latin American Perspective. It provides a synergetic overview on the current state of research in statistics education from a regional perspective. It highlights the specific paradigm of the Latin approach in comparison to international research. For increasing the impact, we publish the papers in English and in the native languages of the authors. This should link those who are the object and subject of the research in their own language to the international trends in research. The aim of the special issue is to increase the awareness of the Latin paradigm and its potential. It will be interesting to see whether a continuous flow between the different paradigms in both directions may also influence future research in the English world.

This special issue recognizes that research in statistics education in Latin American countries has grown remarkably in the last few decades. This phenomenon reflects the need for society to educate statistical literate citizens who are able to use information to make informed decisions - be it as citizens in a democracy or when making economic or health decisions. Today we know so well how important it is to understand statistics and to be statistically literate. This special issue presents to the international community a variety of papers that reveal the epistemological and methodological plurality of research approaches by Latin American scholars. It is essential to disseminate the advances, embedded in cultures that are deeply influenced by closely related languages, across cultures to provide new incentives for international research.

This special issue was very well accepted by the Latin American research community. In the first call, we received more than one hundred abstracts accounting for different areas of research in statistics education. In these abstracts, scholars revealed research interest related to cognitive, motivational, attitudinal, curricular, philosophical, political, and technological processes to understand and promote the teaching and learning of statistics and probability, as well as factors that interfere in the development and understanding of statistical and probabilistic reasoning and thinking. Specifically, all submissions analyzed current or innovative practices in teaching or developed innovative theories for research in statistics 
education. The educational studies were directed at all school levels, including teacher education at the university. From the submitted abstracts, we realized the plurality of Latin American communities as well their partnership with researchers from other parts of the world.

In the end, we accepted 16 interesting and challenging papers from the initial expressions of interest. The authors are from 15 different countries scattered over the Latin world. The papers of this special issue cover a variety of topics such as teaching and learning of statistics and probability, teacher education, professional development, teachers' attitudes, and survey of research in statistics education. Within the teaching and learning topics, researchers focused on different statistical objects such as opinion surveys, statistical graphs and tables, sampling, histograms, variables and statistical knowledge, as well as literacy and thinking. These objects were directed towards a wide range of school levels starting from early childhood education up to college level. The participant groups range from early childhood education to tertiary university education.

The key ideas of the papers in the present issue are diverse and distinctive, with learning within activity-based environments a theme of several papers. Corbo and Sasaki utilized with active-learning methodologies in an introductory course in probability and statistics for engineering at a Brazilian public institution to investigate the impact of collaborative learning arrangements like Jigsaw, Peer Instruction and Think-Pair-Share. The relevance of the two fundamental issues "recognition of need for data" and "statistical thinking" for mathematics teacher professional development are the foci of Gómez-Torres. This research study was carried out with ten in-service Colombian secondary-school mathematics teachers during an educational experience. Oliveira and Reis discuss aspects of statistical literacy in the context of conducting an opinion survey in the perspective of the teaching-learning approach of the Nossa Escola Pesquisa Sua Opinião (Nepso) Program. This empirical research was based on the observation of eight meetings of students (aged 13-14), who focused on the development of an opinion survey. Kataoka, Carvalho Vita and da Silva investigated the support of tactile models in the teaching of chance at the early-childhood level. As a research methodology, they used the theory of semiotic representations to identify different types of semiotic registers and transformations of the participants when working on the tasks in chance contexts.

Scaling the abilities of elementary school pupils with respect to their ability in reading graphs and other representations is the focus of several papers. The topic of the research conducted by Arteaga, Díaz-Levicoy and Batanero is reading levels of line graphs. The authors described common errors and reading levels of Chilean primary-school children (Grades 6 and 7) when working with line graphs. Guimarães, Evangelista and Oliveira explored the abilities of school students to extract data from representations. In their research study, the authors analyzed how far elementary school students (Grades 1 to 5) in the Greater Recife area (Brazil) were able to extract information represented in tables.

Three more theory-oriented papers are included in the issue. One is about conceptual appropriation shaped by collaborative situations, another analyzed attitudes of university students towards statistics, and the third investigated pre-service primary teachers developing investigative cycles for empirical work. The contribution "Discussion circle for the teachinglearning of statistics" from Magalhães and Magalhães puts the focus on university students in Brazil. The authors discuss the conceptual appropriation by means of the creation of collaborative situations in which the argumentative language mediated the construction of knowledge. Abbiati et al. provide an interesting illustration of the research studies from Argentina. They investigated the attitudes of university students towards statistics in introductory courses for non-statisticians (biological sciences, agricultural sciences). With the interest on primary teacher education, Ubilla et al. analyzed the written reports of primary education pre-service teachers when they developed an investigative cycle in a Chilean and a 
Spanish university. The authors observed characteristics of summary research questions and differential features (e.g., the data collection tools and techniques).

Statistics for non-statistics students and understanding of sampling connects two further studies directed towards university and upper secondary level. López and Ramírez investigated university-level students in Mexico. The authors examined programs of introductory-statistics courses with the aim of analyzing and characterizing the state of statistical education for nonstatisticians. The contribution of Reyes and Contreras García takes us into secondary schools in Chile. The authors investigated the understanding of sampling with data, which was collected with an open-ended written questionnaire distributed to secondary students (Grades $8,10 \& 12$ ) in six different schools.

The classroom practice is the object of the next contributions. Gómez-Blancarte, Chávez and Chávez present insights from a one-year project with the intention to characterize the teaching of statistics in Mexican high school education. Guiñez, Vásquez, Brito and Martínez report on the results of a study that examined how Chilean primary-school teachers' attitudes towards probability and its teaching were influenced by their use of an interactive story. This interactive study, "Alice in Randomland", focused on sparking interest in probability and providing opportunities for learning this mathematical topic.

Conceptually oriented are the following papers. The contribution of González takes us to Venezuela to investigate teachers' conceptual and professional knowledge of variability when interpreting histograms in the frame of a case study. Developing data sense is the focus of the contribution by Estrella, Vergara and González. Within the statistical-literacy framework and using a lesson-study approach, a lesson plan for Grade 5 was developed in which Chilean students were encouraged to make inferences from variability in the context of Tsunami data. To conclude the special issue, Da Silva Tinti and Lopes provide a synergetic overview on Brazilian research. The authors analyzed research on statistics education involving teachers to highlight methodological trends and training contexts.

The purpose of this special issue was to gain knowledge from scholars with a Latin language as mother tongue to make visible the peculiarities of their inquiry environment and to learn from their approach, which may have been influenced by their language-specific culture and studies of papers in English, which are influenced by a different paradigm. The intention was, to let the ideas also flow from the Latin embedding to the English world. Throughout the papers, the international community will find a sample of research in statistics education from a Latin-American perspective and will see examples of how cultural factors affect teaching practice and educational research. The papers published in this issue share some similarities in terms of curricular concerns but there is a variety in terms of the epistemological possibilities. Some of the contributing authors have conducted their studies following the classical empirical paradigm of research such as experimental-control design while others have taken a historical-cultural perspective. Thus, the papers published account for cultural diversity and traditions of research.

Looking back, the process to shape the special issue from a Latin American perspective also required some reflections of those outside this culture. After working with the Latin community on this special issue, we have found that there is a strong need to keep supporting scholars from Latin American countries to make their work more widely known for an international audience. Most top journals that publish advances in statistics education require contributions in English, which keeps recurring as a serious limitation since languages spoken in Latin America are mainly Spanish and Portuguese. Opening this journal to the opportunity to publish in two languages, mother tongue and English, is pertinent to encouraging motivated scholars to publish their research results in English but remain rooted in their local cultural environment. Collaboration among expert scholars and international associations will always be welcome to support the intercultural flow and exchange of ideas and approaches. Culture 
per se enriches but requires extra efforts to enable the researchers to benefit from it. One key aspect hereby is the language barrier that needs careful attention and support.

This communication process also raised some general questions to think about in the future. "How can the academic community offer visibility to innovative ways to deal with statistical information that comes from non-academic fields? How can teachers benefit from these ways?" Teachers' expertise might bring us many insights, but they are often bound by their daily duties that leave no margin to reflect, communicate, and network about their ideas, experience problems, and develop ad-hoc approaches to the needs of students in the classroom when learning is under construction. "Is there no time to listen to their voice as protagonists? How could we establish an atmosphere, in which research can link to these key players in education?" These are issues we have to deal with in the future. Whether this issue intensifies the intercultural exchange of ideas is upon the readers. We hope that readers will enjoy the ideas exposed in this special issue on Statistics Education Research from a Latin American Perspective and spread the message.

\section{ACKNOWLEDGEMENTS}

Within the process of shaping this special issue, we experienced the support of many people. Our guest editors, the reviewers, and the assistant editor of SERJ, Noleine Fitzallen, helped us to transfer the ideas from Latin languages to English and to polish the final English versions. Especially, Noleine supported us tremendously in improving the quality of the layout and the English with her intensive feedback. We also thank all reviewers for their support in writing constructive reviews to improve the quality of the expositions and the clarity in writing of the papers. Finally, yet importantly, we are very grateful to all authors. All these papers make this issue a special collection. 